PROCEEDINGS OF THE

AMERICAN MATHEMATICAL SOCIETY

Volume 130, Number 10, Pages 2871-2881

S 0002-9939(02)06437-7

Article electronically published on March 25, 2002

\title{
CONVEXITY NUMBERS OF CLOSED SETS IN $\mathbb{R}^{n}$
}

\author{
STEFAN GESCHKE AND MENACHEM KOJMAN
}

(Communicated by Carl G. Jockusch, Jr.)

\begin{abstract}
For $n>2$ let $\mathcal{I}_{n}$ be the $\sigma$-ideal in $\mathcal{P}\left(n^{\omega}\right)$ generated by all sets which do not contain $n$ equidistant points in the usual metric on $n^{\omega}$. For each $n>2$ a set $S_{n}$ is constructed in $\mathbb{R}^{n}$ so that the $\sigma$-ideal which is generated by the convex subsets of $S_{n}$ restricted to the convexity radical $K\left(S_{n}\right)$ is isomorphic to $\mathcal{I}_{n}$. Thus $\operatorname{cov}\left(\mathcal{I}_{n}\right)$ is equal to the least number of convex subsets required to cover $S_{n}$ - the convexity number of $S_{n}$.

For every non-increasing function $f: \omega \backslash 2 \rightarrow\left\{\kappa \in \operatorname{card}: \operatorname{cf}(\kappa)>\aleph_{0}\right\}$ we construct a model of set theory in which $\operatorname{cov}\left(\mathcal{I}_{n}\right)=f(n)$ for each $n \in$ $\omega \backslash 2$. When $f$ is strictly decreasing up to $n, n-1$ uncountable cardinals are simultaneously realized as convexity numbers of closed subsets of $\mathbb{R}^{n}$. It is conjectured that $n$, but never more than $n$, different uncountable cardinals can occur simultaneously as convexity numbers of closed subsets of $\mathbb{R}^{n}$. This conjecture is true for $n=1$ and $n=2$.
\end{abstract}

\section{INTRODUCTION}

A rich collection of cardinal invariants of the continuum has been studied in modern set theory, yet it is quite rare to find in the literature results on cardinal invariants which are related to the linear structure of $\mathbb{R}^{n}$. One recent exception is the proof by Steprans [8] that covering $R^{n+1}$ by smooth $n$-dimensional surfaces is harder than covering $R^{n+2}$ by smooth $(n+1)$-dimensional surfaces.

In the present paper we investigate invariants of the linear structure of finite and infinite-dimensional Euclidean spaces and their dependence on the dimension. For a closed set $S \subseteq \mathbb{R}^{n}$ consider the $\sigma$-ideal which is generated by the convex subsets of $S$. The interesting case is when this ideal is proper, that is, when $S$ is not countably convex, i.e., not a countable union of convex sets. In this case, the ideal contains a maximal open subset whose complement in $S$ is a perfect set, denoted by $K(S)$. The restriction of the $\sigma$-ideal to $K(S)$ is denoted by $\mathcal{I}(S)$ and is contained in the meager ideal on $K(S)$. Therefore, the least number of convex subsets of $S$ required to cover $S$, called the convexity number of $S$ and denoted by $\gamma(S)$, is at least as large as $\operatorname{cov}(\mathcal{M})$ - the covering number of meager sets.

There are plenty of simply defined sub-ideals of the meager ideal and, as shown in 4], uncountably many different cardinals can be simultaneously realized as covering numbers of simple sub-ideals of the meager ideal, but all of this does not imply that

Received by the editors April 19, 2001 and, in revised form, May 31, 2001.

1991 Mathematics Subject Classification. Primary 05A20, 52A05, 03E17, 03E35; Secondary $03 \mathrm{E} 75$.

Key words and phrases. Convex cover, convexity number, $n$-space, forcing extension, covering number.

(C)2002 American Mathematical Society 
there are many ideals that may occur as $\mathcal{I}(S)$ for a closed $S \subseteq \mathbb{R}^{n}$. It is an easy exercise to show that if $S \subseteq \mathbb{R}^{1}$, then $\mathcal{I}(S)$ is at most the ideal of countable sets. In [3] it was shown that, except for the ideal of countable sets, essentially only one type of meager ideal can occur as $\mathcal{I}(S)$ for a closed $S \subseteq \mathbb{R}^{2}$, that the covering number of this type of ideal can consistently be smaller than $\mathfrak{c}$, and that an uncountable convexity number $\gamma=\gamma(S)<2^{\aleph_{0}}$ of a closed $S \subseteq \mathbb{R}^{2}$ must satisfy $\gamma^{+}=2^{\aleph_{0}}$. Thus exactly 1 uncountable convexity number of a closed set can be realized in $\mathbb{R}^{1}$ and 2 but not more than 2 uncountable convexity numbers of closed sets can be realized in $\mathbb{R}^{2}$.

At present we have no evidence that more than $n$ different uncountable cardinals may simultaneously occur as convexity numbers of closed subsets of $\mathbb{R}^{n}$. Let $\sigma_{\text {conv }}\left(\mathbb{R}^{n}\right)$ denote the set of all uncountable convexity numbers of closed subsets of $\mathbb{R}^{n}$. In 3] it was conjectured that for every $n \in \omega,\left|\sigma_{\text {conv }}\left(\mathbb{R}^{n}\right)\right| \leq n$ but that for each $n$ (separately) $\left|\sigma_{\text {conv }}\left(\mathbb{R}^{n}\right)\right|=n$ is consistent. For $n=1$ and $n=2$ this is indeed true.

In the present paper we define closed subsets $S_{n} \subseteq \mathbb{R}^{n}$ for $n>2$ so that $\mathcal{I}\left(S_{n}\right)$ is equal to the ideal $\mathcal{I}_{n}$ introduced by Newelski and Roslanowski in [6]. The ideal $\mathcal{I}_{n}$ is generated over the space $n^{\omega}$ by all sets which do not contain an equidistant $n$-tuple with respect to the usual metric. Equivalently, regarding $n^{\omega}$ as a tree, which is more convenient for what follows, the ideal $\mathcal{I}_{n}$ is generated by all closed sets which are coded by $<n$ splitting subtrees of $n^{\omega}$.

Newelski and Roslanowski have proved the consistency of $\operatorname{cov}\left(\mathcal{I}_{n+1}\right)<\operatorname{cov}\left(\mathcal{I}_{n}\right)$. We extend their result by showing that $\operatorname{cov}\left(\mathcal{I}_{i}\right)=f(i)$ is consistent simultaneously for all $i<n$ where $f$ is any non-increasing function from $n$ to the cardinals with uncountable cofinality. A parallel result was obtained by Shelah and Steprans in [7] for similar looking ideals using a different method. When choosing $f$ to be strictly decreasing, the following is obtained.

Theorem 1.1. For each $n>0,\left|\sigma_{\text {conv }}\left(\mathbb{R}^{n}\right)\right| \geq n-1$ is consistent.

\subsection{Notation.}

Definition 1.2. A set $T \subseteq \omega^{<\omega}$ is a tree if $T$ is closed with respect to restrictions to natural numbers. For a tree $T$ and $t \in T$ let $\operatorname{succ}_{T}(t):=\{s \in T: \exists i \in \omega(s=t \frown i)\}$ where $\frown$ is the concatenation of sequences and $i$ is identified with the sequence of length one with value $i$. For $n \in \omega, T$ is $<n$-ary if for all $t \in T$, $\left|\operatorname{succ}_{T}(t)\right|<n$. For $\alpha \leq \omega$ and a set $X \subseteq \omega^{\alpha}$ let

$$
T(X):=\left\{t \in \omega^{<\omega}: \exists x \in X(t \subseteq x)\right\} .
$$

$X$ is $<n$-ary if $T(X)$ is. For $n \in \omega$ let $\mathcal{I}_{n}$ be the ideal of $<n$-ary subsets of $n^{\omega}$.

\section{Closed subsets of $\mathbb{R}^{n}$ With PREscribed CONVEXity ideAls}

In this section, for each $n \in \omega \backslash 2$ we construct a closed subset $X$ of $\mathbb{R}^{n}$ with $\gamma(X)=\operatorname{cov}\left(\mathcal{I}_{n}\right)$.

Fix $n \in \omega \backslash 2$. Let $B^{n}$ denote the closed unit ball in $\mathbb{R}^{n}$ and let $S^{n}$ denote the boundary of $B^{n}$. In order to get $X$, we construct a homeomorphism $h$ between $n^{\omega}$ and a subset $Y$ of $S^{n}$ with nice properties. $X$ is then defined as a subset of the convex hull of $Y$ such that the convex hull of a subset of $Y$ is included in $X$ if and only if the set corresponds to a $<n$-ary subset of $n^{\omega}$. $Y$ is the set of accumulation points of a family $\left(x_{s}\right)_{s \in n<\omega}$ constructed in the next lemma. 
For a subset $S$ of a linear space let $\operatorname{conv}(S)$ be the convex hull of $S$.

For a point $x \in \operatorname{int}\left(B^{n}\right) \backslash\{0\}$ let $P(x)$ denote the hyperplane containing $x$ which is orthogonal to the line through $x$ and 0 . Let $H(x)$ denote the halfspace in $\mathbb{R}^{n}$ with boundary $P(x)$ that does not contain 0 . Finally let $C(x)$ denote $H(x) \cap B^{n}$ and let $L(x)$ denote the closed line segment between $x$ and $\frac{1}{\|x\|} x$.

Lemma 2.1. There is a family $\left(x_{s}\right)_{s \in n}<\omega$ of points in $\operatorname{int}\left(B^{n} \backslash\{0\}\right)$ with the following properties:

(1) For all $s, t \in n^{<\omega}$ with $s \subseteq t, C\left(x_{t}\right) \subseteq C\left(x_{s}\right)$.

(2) For all $s, t \in n^{<\omega}$, if $s$ and $t$ are incomparable, then $C\left(x_{s}\right)$ and $C\left(x_{t}\right)$ are disjoint.

(3) For all $m \in \omega$ and all $t \in n^{m}$ the diameter of $C\left(x_{t}\right)$ is less than $2^{-m}$.

(4) For all $s \in n^{<\omega}$ and all $z_{i} \in C\left(x_{s} \frown i\right), i<n, \operatorname{conv}\left(\left\{z_{i}: i<n\right\}\right)$ intersects $L\left(x_{s}\right)$.

(5) For all $m \in \omega$ and all $t \in n^{m}$, if $S \subseteq n^{m+1}$ is such that

$$
\left|\left\{i \in n: t^{\frown} i \in S\right\}\right|<n,
$$

then $\operatorname{conv}\left(\bigcup_{s \in S} C\left(x_{s}\right)\right)$ is disjoint from $L\left(x_{t}\right)$.

Proof. We construct the family $\left(x_{s}\right)_{s \in n<\omega}$ by recursion. Start with any point $x_{\emptyset} \in$ $\operatorname{int}\left(B^{n}\right) \backslash\{0\}$ for which the diameter of $C\left(x_{\emptyset}\right)$ is less than 1 . Let $m \in \omega$ and suppose $x_{s}$ has been defined for all $s \in n^{m}$ and we want to define $x_{t}{ }_{i}, i<n$, for a fixed $t \in n^{m}$

$P\left(x_{t}\right) \cap B^{n}$ is an $n$-1-dimensional ball centered at $x_{t}$. There are pairwise distinct points $y_{0}, \ldots, y_{n-1} \in P\left(x_{t}\right) \cap B_{n}$ such that relative to the affine subspace $P\left(x_{t}\right)$ of $\mathbb{R}^{n}, x_{t}$ is contained in the interior of $\operatorname{conv}\left(\left\{y_{0}, \ldots, y_{n-1}\right\}\right)$, but not in the convex hull of any proper subset of $\left\{y_{0}, \ldots, y_{n-1}\right\}$.

Now, for

$$
S \subseteq\left\{y_{0}, \ldots, y_{n-1}\right\} \cup \bigcup\left\{C\left(x_{s}\right): s \in n^{m} \wedge s \neq t\right\}
$$

$\operatorname{conv}(S)$ and $L\left(x_{t}\right)$ are disjoint if and only if

$$
\left|\left\{y_{0}, \ldots, y_{n-1}\right\} \cap S\right|<n .
$$

But this remains true if we replace each $y_{i}$ by any element of $\operatorname{int}\left(C\left(x_{t}\right)\right)$ which is sufficiently close to $y_{i}$. It follows that we can find $x_{t-i} \in \operatorname{int}\left(C\left(x_{t}\right)\right), i<n$, such that for all $S \subseteq \bigcup\left\{C\left(x_{s}\right): s \in n^{m} \wedge s \neq t\right\} \cup \bigcup_{i<n} C\left(x_{t}-i\right)$ we have

$$
\left|\left\{i<n: C\left(x_{t} \frown i\right) \cap S \neq \emptyset\right\}\right|<n
$$

if and only if $\operatorname{conv}(S)$ is disjoint from $L\left(x_{t}\right)$. Moreover, we can chose $x_{t-i}, i<n$, such that:

(i) for all $i, j<n$ with $i \neq j, C\left(x_{t-i}\right)$ is disjoint from $C\left(x_{t-j}\right)$ and

(ii) for all $i<n, C\left(x_{t}-i\right)$ is included in $C\left(x_{t}\right)$ and has diameter less than $2^{-(m+1)}$.

(i) can be accomplished by choosing $x_{t-i}$ sufficiently close to $y_{i}$ and (ii) by choosing $x_{t-i}$ sufficiently close to $S^{n}$.

Proceeding in this way, we get a family $\left(x_{s}\right)_{s \in n<\omega}$ with the desired properties.

Using Lemma 2.1] we can show

Theorem 2.2. For each $n \in \omega \backslash 2$ there is a closed subset of $\mathbb{R}^{n}$ such that $\gamma(X)=$ $\operatorname{cov}\left(\mathcal{I}_{n}\right)$. 
Proof. Let $\left(x_{s}\right)_{s \in n<\omega}$ be as in Lemma 2.1 Let $Y$ be the set of accumulation points of $\left\{x_{s}: s \in n^{<\omega}\right\}$. Clearly, $Y \subseteq S^{n}$.

For each $\sigma \in n^{\omega}$ let $h(\sigma)$ be the unique element of $\bigcap_{m \in \omega} C\left(x_{\sigma\lceil m}\right)$. Note that $h: n^{\omega} \rightarrow Y$ is a homeomorphism. We define a first approximation $\tilde{X}$ of $X$.

For $m \in \omega$ let

$$
\tilde{X}_{m}=\bigcup\left\{\operatorname{conv}\left(\bigcup\left\{C\left(x_{s}\right): s \in I\right\}\right): I \subseteq n^{m} \text { is }<n \text {-ary }\right\} .
$$

Clearly, each $\tilde{X}_{m}$ is closed. Let $\tilde{X}:=\bigcap_{m \in \omega} \tilde{X}_{m}$.

Note that if $S \subseteq n^{\omega}$ is $<n$-ary, then $\operatorname{conv}(h[S])$ is included in every $\tilde{X}_{m}, m \in \omega$. This shows part a) of

Claim 2.3. Let $S \subseteq n^{\omega}$. Then:

a) If $S$ is $<n$-ary, then $\operatorname{conv}(h[S]) \subseteq \tilde{X}$.

b) $\tilde{X}$ is disjoint from each $L\left(x_{s}\right), s \in n^{<\omega}$.

Part b) of this claim follows from the fact that by property (5) in Lemma 2.1 for all $m \in \omega$ and all $s \in n^{m}, \tilde{X}_{m}$ is disjoint from $L\left(x_{s}\right)$.

Since $\tilde{X}$ is closed and the length of each $L\left(x_{s}\right)$ is strictly less than $2^{-\operatorname{dom}(s)}$, for each $s \in n^{<\omega}$ there is an open set $U_{s}$ such that:

(i) $L\left(x_{s}\right) \subseteq U_{s}$,

(ii) $U_{s}$ is disjoint from $\tilde{X}$,

(iii) the diameter of $U_{s}$ is less than $2^{-\operatorname{dom}(s)}$, and

(iv) $B^{n} \backslash U_{s}$ is countably convex.

For (iv) it is sufficient to choose $U_{s}$ as the interior of a polyhedron.

Let

$$
X:=\operatorname{conv}(Y) \backslash \bigcup\left\{U_{s}: s \in n^{<\omega}\right\} .
$$

It is clear that $X$ is closed.

Claim 2.4. $S \subseteq n^{\omega}$ is $<n$-ary if and only if $\operatorname{conv}(h[S])$ is included in $X$.

Since $\tilde{X} \subseteq X$, by Claim 2.3, for every $<n$-ary set $S \subseteq n^{\omega}, \operatorname{conv}(h[S]) \subseteq X$. On the other hand, if $S \subseteq n^{\omega}$ is not $<n$-ary, then there are $\sigma_{0}, \ldots, \sigma_{n-1} \in S$ and there is $t \in n^{<\omega}$ such that for $m:=\operatorname{dom}(t),\left\{\sigma_{i}\lceil m+1: i<n\}=\left\{t^{\frown} i: i<n\right\}\right.$. By property (4) in Lemma 2.1, $\operatorname{conv}\left(\left\{h\left(\sigma_{i}\right): i<n\right\}\right)$ intersects $L\left(x_{t}\right)$. However, $X$ is disjoint from $L\left(x_{t}\right)$. Therefore $\operatorname{conv}(h[S])$ is not included in $X$. This shows Claim 2.4.

Now, whenever $\mathcal{D}$ is a collection of convex subsets of $X$, for each $D \in \mathcal{D}, h^{-1}[D]$ is $<n$-ary. $\mathcal{D}$ covers $Y$ if and only if $\left\{h^{-1}[D]: D \in \mathcal{D}\right\}$ covers $n^{\omega}$. It follows that the minimal size of a family of convex subsets of $X$ covering $Y$ is exactly $\operatorname{cov}\left(\mathcal{I}_{n}\right)$. Thus we are done if we can show

Claim 2.5. $X \backslash Y$ is countably convex.

First note that $Y=X \cap S^{n}$. Now fix a countable base $\mathcal{V}$ for the open sets in $\mathbb{R}^{n}$ consisting of convex sets. For each $x \in X \backslash Y$ there is $V_{x} \in \mathcal{V}$ such that $x \subseteq V_{x} \subseteq B^{n}$ and $V_{x}$ intersects only finitely many of the $U_{s}, s \in n^{<\omega} . V_{x} \cap X$ is the intersection of finitely many countably convex sets. Therefore $V_{x} \cap X$ itself is countably convex. But since $\mathcal{V}$ is countable, the set $\left\{V_{x} \cap X: x \in X \backslash Y\right\}$ is countable. It follows that $X \backslash Y=\bigcup\left\{V_{x} \cap X: x \in X \backslash Y\right\}$ is countably convex. 
2.1. An infinite-dimensional example. The sets $X \subseteq \mathbb{R}^{n}$ constructed above have an infinite-dimensional analogue.

Definition 2.6. Let $\mathcal{I}_{\omega}$ be the ideal on $\prod_{n \in \omega}(n+1)$ consisting of those sets $S$ such that for each $t \in T(S)$ with $t \neq \emptyset$, $\left|\operatorname{succ}_{T(S)}(t)\right| \leq \operatorname{dom}(t)$. Note that for each nonempty $t \in T\left(\prod_{n \in \omega}(n+1)\right)$ we have $\left|\operatorname{succ}_{T\left(\prod_{n \in \omega}(n+1)\right)}(t)\right|=\operatorname{dom}(t)+1$. Let $T_{\omega}:=T\left(\prod_{n \in \omega}(n+1)\right)$.

Note that $T_{\omega}=\bigcup_{n \in \omega} \prod_{m<n}(m+1)$.

Let $V$ be an infinite-dimensional Hilbert space and let $B$ denote the unit ball in $H$. For each point $x \in \operatorname{int}(B) \backslash\{0\}$ let $P(x), H(x), C(x)$, and $L(x)$ be defined as in the finite-dimensional case. A slight modification of the proof of Lemma 2.1 gives

Lemma 2.7. There is a family $\left(x_{s}\right)_{s \in T_{\omega}}$ of points in $\operatorname{int}(B \backslash\{0\})$ with the following properties:

(1) For all $s, t \in T_{\omega}$ with $s \subseteq t, C\left(x_{t}\right) \subseteq C\left(x_{s}\right)$.

(2) For all $s, t \in T_{\omega}$, if $s$ and $t$ are incomparable, then $C\left(x_{s}\right)$ and $C\left(x_{t}\right)$ are disjoint.

(3) For all $t \in T_{\omega}$ the diameter of $C\left(x_{t}\right)$ is less than $2^{-d o m(t)}$.

(4) For all $n \in \omega$, all $s \in \prod_{m<n}(m+1)$ and all $z_{i} \in C\left(x_{s-i}\right), i<n+1$, $\operatorname{conv}\left(\left\{z_{i}: i<n+1\right\}\right)$ intersects $L\left(x_{s}\right)$.

(5) For all $n \in \omega$ and all $t \in \prod_{m<n}(m+1)$, if $S \subseteq \prod_{m<n+1}(m+1)$ is such that

$$
\mid\{i<n+1: t \frown i \in S)\} \mid<n+1,
$$

then $\operatorname{conv}\left(\bigcup_{s \in S} C\left(x_{s}\right)\right)$ is disjoint from $L\left(x_{t}\right)$.

Using this lemma and a straightforward modification of the proof of Theorem 2.2 we obtain:

Theorem 2.8. For every infinite-dimensional Hilbert space $V$ there is a closed subset of $H$ such that $\gamma(X)=\operatorname{cov}\left(\mathcal{I}_{\omega}\right)$.

\section{INEQUALITIES IN ZFC}

As mentioned before, it has been shown in [6] that $\operatorname{cov}\left(\mathcal{I}_{m}\right)<\operatorname{cov}\left(\mathcal{I}_{n}\right)$ is consistent for $n<m$. We cannot hope for more independence results here since $n<m$ implies $\operatorname{cov}\left(\mathcal{I}_{m}\right) \leq \operatorname{cov}\left(\mathcal{I}_{n}\right)$ in ZFC.

Lemma 3.1. a) $\operatorname{cov}\left(\mathcal{I}_{m}\right) \leq \operatorname{cov}\left(\mathcal{I}_{n}\right)$ for all $n, m \in \omega \backslash 2$ with $n<m$.

b) $\operatorname{cov}\left(\mathcal{I}_{\omega}\right) \leq \operatorname{cov}\left(\mathcal{I}_{n}\right)$ for all $n \in \omega \backslash 2$.

Proof. For a) let $n, m \in \omega \backslash 2$ be such that $n<m$. Let $f: m \rightarrow n$ be onto. $f$ induces a continuous map $g: m^{\omega} \rightarrow n^{\omega}$ which is onto. It is easily seen that the preimage of a $<n$-ary subset of $n^{\omega}$ under $g$ is a $<m$-ary subset of $m^{\omega}$. It follows that for a family $\mathcal{F}$ of $<n$-ary subsets of $n^{\omega}$ which covers $n^{\omega},\left\{g^{-1}[F]: F \in \mathcal{F}\right\}$ is a family of $<m$-ary subsets of $m^{\omega}$ which covers $m^{\omega}$. This implies $\operatorname{cov}\left(\mathcal{I}_{m}\right) \leq \operatorname{cov}\left(\mathcal{I}_{n}\right)$.

The proof of $b$ ) is similar.

The cardinal invariant $\mathfrak{h m}$ turns out to be a natural upper bound for $\operatorname{cov}\left(\mathcal{I}_{n}\right)$, $n>2$. Recall the definition of $\mathfrak{h m}$. A set $S \subseteq 2^{\omega}$ is monochromatic if for all unordered pairs $\{x, y\} \in\left[2^{\omega}\right]^{2}$ the parity of the first coordinate where $x$ and $y$ disagree is the same. $\mathfrak{h m}$ is the covering number of the ideal of monochromatic subsets of $2^{\omega}$. 
Lemma 3.2. $\operatorname{cov}\left(\mathcal{I}_{n}\right) \leq \mathfrak{h m}$ for all $n \in \omega \backslash 3$.

Proof. By Lemma 3.1 it is sufficient to $\operatorname{show} \operatorname{cov}\left(\mathcal{I}_{3}\right) \leq \mathfrak{h m}$.

Let $S \subseteq 2^{\omega}$. Then $S$ is monochromatic if and only if $T(S)$ branches only at even levels or only at odd levels. We define a homeomorphism $h: 2^{\omega} \rightarrow 3^{\omega}$ mapping monochromatic sets onto $<3$-ary sets.

$h$ will be constructed from $f: 3^{<\omega} \rightarrow 2^{<\omega}$ which we define by recursion. Let $f(\emptyset):=\emptyset$. Suppose $f(t)$ has been defined for some $t \in 3^{<\omega}$. For $i<3$ let

$$
f\left(t^{\frown} i\right):= \begin{cases}f(t) \frown 0 & \text { if } i=0, \\ f(t) \frown 1 \frown 0 & \text { if } i=1, \text { and } \\ f(t) \frown 1 \frown 1 & \text { if } i=2 .\end{cases}
$$

For $x$ in $2^{\omega}$ let $h(x):=\bigcup\left\{s \in 3^{<\omega}: f(s) \subseteq x\right\}$.

Note that if $S \subseteq 2^{\omega}$ is monochromatic, then for each $t \in 3^{<\omega}$ at most two of the sequences $f\left(t^{\frown} i\right), i<3$, are contained in $T(S)$. This implies that $h[S]$ is $<3$-ary if $S \subseteq 2^{\omega}$ is monochromatic.

Since $h$ is a bijection, it follows that $\operatorname{cov}\left(\mathcal{I}_{3}\right)$ is not greater than $\mathfrak{h m}$.

We now look for lower bounds of $\operatorname{cov}\left(\mathcal{I}_{\omega}\right)$. Let $\mathcal{M}$ denote the ideal of meager subsets of $2^{\omega}$ and let $\mathcal{N}$ denote the ideal of measure zero subsets of $2^{\omega}$. Recall that for $\operatorname{cov}(\mathcal{M})$ and $\operatorname{cov}(\mathcal{N})$ it does not matter whether we consider the ideals $\mathcal{M}$ and $\mathcal{N}$ on $2^{\omega}$, on $n^{\omega}$ for some $n>2$, on $\prod_{n \in \omega}(n+1)$, or on $\mathbb{R}$.

It is easy to see that every set $S \in \mathcal{I}_{\omega}$ is nowhere dense and of measure zero in $\prod_{n \in \omega}(n+1)$. This implies:

Lemma 3.3. $\max (\operatorname{cov}(\mathcal{M}), \operatorname{cov}(\mathcal{N})) \leq \operatorname{cov}\left(\mathcal{I}_{\omega}\right)$.

\section{Consistency Results}

4.1. The basic forcing notions. We introduce the basic building blocks for our forcing construction. The forcing notions $\mathbb{D}_{n}$ defined below were introduced and studied in [6]. We will use a countable support product of the $\mathbb{D}_{n}$ to construct the models announced above.

Definition 4.1. For each $n \in \omega \backslash 2$ let $\mathbb{D}_{n}$ be the set of trees $p \subseteq n^{<\omega}$ such that for each $t \in p,\left|\operatorname{succ}_{p}(t)\right| \in\{1, n\}$ and there is $s \in T$ such that $t \subseteq s$ and $\left|\operatorname{succ}_{p}(s)\right|=n$. The order on $\mathbb{D}_{n}$ is set inclusion. For $m \in \omega$ and $p \in \mathbb{D}_{n}$ let $p^{m}$ consist of those $t \in p$ such that $\left|\operatorname{succ}_{p}(t)\right|=n$ and $t$ has exactly $m$ proper initial segments with the same property. For $p, q \in \mathbb{D}_{n}$ let $p \leq_{m} q$ if $p \leq q$ and $p^{m}=q^{m}$. A sequence $\left(p_{m}\right)_{m \in \omega}$ in $\mathbb{D}_{n}$ is a fusion sequence if there is a non-decreasing unbounded function $f: \omega \rightarrow \omega$ such that for each $m \in \omega, p_{m+1} \leq_{f(m)} p_{m}$. If $\left(p_{m}\right)_{m \in \omega}$ is a fusion sequence, its fusion $p_{\omega}:=\bigcap_{m \in \omega} p_{m}$ is easily seen to be an element of $\mathbb{D}_{n}$.

Let $p \in \mathbb{D}_{n}$. Each $\sigma \in n^{m}$ uniquely determines an element $t_{\sigma}$ of $p^{m}$. Let $p_{\sigma}:=\left\{s \in p: t_{\sigma} \subseteq s \vee s \subseteq t_{\sigma}\right\}$.

Note that $\mathbb{D}_{2}$ is just the ordinary Sacks forcing and $\mathbb{D}_{n}$ adds a real which is not contained in any $<n$-ary subset of $n^{\omega}$ coded in the ground model. Using a standard fusion argument, one can show that the forcing $\mathbb{D}_{n}$ has the $n$-localization property. That is, any $x \in \omega^{\omega}$ in a generic extension by $\mathbb{D}_{n}$ is contained in a $<n$-ary set coded in the ground model. 
4.2. Making the continuum big. We show how to get various values for $\operatorname{cov}\left(\mathcal{I}_{n}\right)$. This section owes much to [6]. We need a lot of notation.

Definition 4.2. Let $\left(\mathbb{Q}_{i}\right)_{i \in I}$ be a family of forcing notions of the type $\mathbb{D}_{n}$. For each $i \in I$ let $n(i)$ be such that $\mathbb{Q}_{i}=\mathbb{D}_{n(i)}$. For $p \in \prod_{i<I} \mathbb{Q}_{i}$ let supt $p:=\{i \in I$ : $\left.p(i) \neq 1_{\mathbb{Q}_{i}}\right\}$. Let

$$
\mathbb{Q}:=\left\{p \in \prod_{i \in I} \mathbb{Q}_{i}:|\operatorname{supt}(p)| \leq \aleph_{0}\right\}
$$

be the countable support product over the family $\left(\mathbb{Q}_{i}\right)_{i \in I} . \mathbb{Q}$ is ordered coordinatewise. For a finite set $F \subseteq \kappa$ and $\eta: F \rightarrow \omega$ let the relation $\leq_{F, \eta}$ on $\mathbb{Q}$ be defined as follows:

For all $\mathbb{Q}$ let $p \leq_{F, \eta} q$ if $p \leq q$ and for all $i \in F, p(i) \leq_{\eta(i)} q(i)$. For $p, F$, and $\eta$ as before and $\sigma \in \prod_{i \in F} n(i)^{\eta(i)}$ let $p * \sigma$ be such that for all $i \in F,(p * \sigma)(i)=p(i)_{\sigma}$ and for all $i \in I \backslash F,(p * \sigma)(i)=p(i)$.

A sequence $\left(p_{n}\right)_{n \in \omega}$ of conditions in $\mathbb{Q}$ is a fusion sequence if there is an increasing sequence $\left(F_{n}\right)_{n \in \omega}$ of finite subsets of $\kappa$ and a sequence $\left(\eta_{n}\right)_{n \in \omega}$ such that for all $n \in \omega, \eta_{n}: F_{n} \rightarrow \omega$, for all $i \in F_{n}$ we have $\eta_{n}(i) \leq \eta_{n+1}(i), p_{n+1} \leq_{F_{n}, \eta_{n}} p_{n}$, and for all $i \in \operatorname{supt}\left(p_{n}\right)$ there is $m \in \omega$ such that $i \in F_{m}$ and $\eta_{m}(i) \geq n$. If $\left(p_{n}\right)_{n \in \omega}$ is a fusion sequence in $\mathbb{Q}$, then for each $i \in I,\left(p_{n}(i)\right)_{n \in \omega}$ is a fusion sequence in $\mathbb{Q}_{i}$ or constant with value $1_{\mathbb{Q}_{i}}$. This shows that

$$
p_{\omega}:=\left(\bigcap_{n \in \omega} p_{n}(i)\right)_{i \in I}
$$

is a condition in $\mathbb{Q}$, the fusion of the sequence $\left(p_{n}\right)_{n \in \omega}$.

Now let $f: \omega \backslash 2 \rightarrow\left\{\kappa \in\right.$ Card $\left.: \operatorname{cf}(\kappa)>\aleph_{0}\right\}$ be a non-increasing function. We define a forcing notion $\mathbb{P}$ as follows:

Let $J:=\{n \in \omega: \neg \exists m<n(f(n)=f(m))\}$. Let $I$ be a set of size $f(2)$ and let $g: I \rightarrow J$ be a function such that for each $n \in J,\left|g^{-1}(n)\right|=f(n)$. For each $i \in I$ let $\mathbb{Q}_{i}:=\mathbb{D}_{g(i)}$. Finally, let $\mathbb{P}$ be the countable support product over the sequence $\left(\mathbb{Q}_{i}\right)_{i \in I}$.

For each $n \in J$ let $I_{n}:=g^{-1}(n)$ and $I_{\geq n}:=g^{-1}[\omega \backslash n]$. Let $\mathbb{P}_{n}$ be the countable support product over $\left(\mathbb{Q}_{i}\right)_{i \in I_{n}}$ and let $\mathbb{P}_{\geq n}$ be the countable support product over $\left(\mathbb{Q}_{i}\right)_{i \in I_{\geq n}}$ We regard the $\mathbb{Q}_{i}$ 's, $\mathbb{P}_{n}$ 's, and $\mathbb{P}_{\geq n}$ 's as complete suborders of $\mathbb{P}$ in the natural way. Note that $\mathbb{P}=\mathbb{P}_{\geq 2}$.

Lemma 4.3. For each $\mathbb{P}$-name $\dot{x}$ for a new element of $\omega^{\omega}$, every condition $p \in \mathbb{P}$, and each $n \in \omega$ there is a $\mathbb{P}_{\geq n}$-name $\dot{T}$ for an $<n$-ary subtree of $\omega^{<\omega}$ and a condition $q \leq p$ such that $q$ forces $\dot{x}$ to be a branch through $\dot{T}$.

Proof. Let $\dot{x}, p$, and $n$ be as above. We construct a tree $S$ in the ground model and a condition $q \leq p$ which forces that $\dot{x}$ is a branch through $S$. If $G$ is $\mathbb{P}_{\geq n}$-generic over the ground model, we find a $<n$-ary subtree $T$ of $S$ such that $\dot{x}$ is forced to be a branch through $T$. $\dot{T}$ will be the $\mathbb{P}_{\geq n}$-name for $T$.

For a condition $r \in \mathbb{P}$ let $x_{r}$ be the maximal element $t$ of $\omega^{<\omega}$ such that $r \Vdash t \subseteq \dot{x}$. $x_{r}$ exists since $\dot{x}$ is a name for a new real. Let

$$
T_{r}:=\left\{t \in \omega^{<\omega}: \exists r^{\prime}<r\left(r^{\prime} \Vdash t \subseteq \dot{x}\right)\right\}
$$


be the tree of $r$-possibilities for $\dot{x}$. Clearly, $r$ forces $\dot{x}$ to be a branch through $T_{r}$. The ground model tree $S$ will be $T_{q}$. We have to find $q$.

For $s, t \in \omega^{<\omega}$ we write $s \perp t$ if $s$ and $t$ are incomparable. Let $F \in[I]^{<\aleph_{0}}$ and $\eta: F \rightarrow \omega$. A condition $r \in \mathbb{P}$ is $(F, \eta)$-faithful if for all $r^{\prime} \leq_{F, \eta} r$ and all $\sigma, \tau \in \prod_{i \in F} g(i)^{\eta(i)}$ with $x_{r_{\sigma}^{\prime}} \perp x_{r_{\tau}^{\prime}}$ already $x_{r_{\sigma}} \perp x_{r_{\tau}}$.

Claim 4.4. Suppose $r$ is $(F, \eta)$-faithful.

a) Let $j \in I \backslash F$ and define $F^{\prime}:=F \cup\{j\}$ and $\eta^{\prime}:=\eta \cup\{(j, 0)\}$. Then $r$ is $\left(F^{\prime}, \eta^{\prime}\right)$-faithful.

b) Suppose $r$ is $(F, \eta)$-faithful. Let $j \in F$, and let $\eta^{\prime}$ be such that for all $i \in$ $F \backslash\{j\}, \eta^{\prime}(i)=\eta(i)$ and $\eta^{\prime}(j)=\eta(j)+1$. Then there is a condition $r^{\prime} \leq_{F, \eta} r$ such that $r^{\prime}$ is $\left(F, \eta^{\prime}\right)$-faithful.

Part a) of this claim follows immediately from the definitions. For the proof of b) fix an enumeration $\left\{\sigma_{1}, \ldots, \sigma_{z}\right\}$ of $\prod_{i \in F} g(i)^{\eta(i)}$. We define a $\leq_{F, \eta^{-} \text {-descending }}$ sequence $\left(r_{k}\right)_{k<z}$ below $r$.

For $k \in\{1, \ldots, z\}$ and $l<g(j)$ let $\sigma_{k}^{l}$ be such that for all $i \in F \backslash\{j\}, \sigma_{k}^{l}(i)=\sigma_{k}(i)$ and $\sigma_{k}^{l}(j)=\sigma_{k}(j) \frown l$. Let $r_{0}:=r$.

Suppose we have constructed $r_{k}$ for some $k<z$ and we want to build $r_{k+1}$. Let $r_{k+1} \leq_{F, \eta^{\prime}} r_{k}$ be such that the set $\left\{x_{r_{k+1} * \sigma_{k+1}^{l}}: l<g(j)\right\}$ has the largest possible cardinality.

Let $r^{\prime}:=r_{z}$.

Subclaim. $r^{\prime}$ is $\left(F, \eta^{\prime}\right)$-faithful.

Suppose not. Then there is $r^{\prime \prime} \leq_{F, \eta^{\prime}} r^{\prime}$ such that for some $\tau, \rho \in \prod_{i \in F} g(i)^{\eta^{\prime}(i)}$ we have $x_{r_{\tau}^{\prime \prime}} \perp x_{r_{\rho}^{\prime \prime}}$, but $x_{r_{\tau}^{\prime}} \not \perp x_{r_{\rho}^{\prime}}$. Since $r$ is $(F, \eta)$-faithful and $r^{\prime} \leq_{F, \eta} r$, this implies that there is $k \in\{1, \ldots, z\}$ such that for some $l_{0}, l_{1}<g(j)$ with $l_{0} \neq l_{1}$, $\tau=\sigma_{k}^{l_{0}}$ and $\rho=\sigma_{k}^{l_{1}}$.

But now $\left\{x_{r^{\prime \prime} * \sigma_{k}^{l}}: l<g(j)\right\}$ has a larger cardinality than $\left\{x_{r_{k} * \sigma_{k}^{l}}: l<g(j)\right\}$, contradicting the choice of $r_{k}$. This shows the subclaim and finishes the proof of b).

Using a) and b) of Claim 4.4 together with some bookkeeping, we construct a sequence $\left(p_{m}, F_{m}, \eta_{m}, j_{m}\right)_{m \in \omega}$ such that:

(1) $\left(p_{m}\right)_{m \in \omega}$ is a fusion sequence in $\mathbb{P}$ witnessed by $\left(F_{m}, \eta_{m}\right)_{m \in \omega}$ and $F_{0}=\eta_{0}=\emptyset$,

(2) for all $m \in \omega, p_{m}$ is $\left(F_{m}, \eta_{m}\right)$-faithful and $p_{m} \leq p$,

(3) for all $m \in \omega$ and all $i \in F_{m}, \eta_{m+1}(i) \leq \eta_{m}(i)+1$,

(4) for all $m \in \omega, j_{m}$ is the unique element of $F_{m+1}$ such that either $j_{m} \in F_{m}$ and $\eta_{m+1}\left(j_{m}\right)=\eta_{m}\left(j_{m}\right)+1$ or $j_{m} \notin F_{m}$ and $\eta_{m+1}\left(j_{m}\right)=1$, and

(5) for all $m \in \omega$ and all $\sigma \in \prod_{i \in F_{m}} g(i)^{\eta_{m}(i)}, p_{m} * \sigma$ decides at least $\dot{x}\lceil m$.

Let $q$ be the fusion of the $p_{m}, m \in \omega$, and let $S:=T_{q}$. Note that for all $m \in \omega$, $q \leq_{F_{m}, \eta_{m}} p_{m}$. For each $m \in \omega$ let $S_{m+1}$ be the tree generated by

$$
\left\{x_{p_{m} * \sigma}: \sigma \in \prod_{i \in F_{m}} g(i)^{\eta_{m}(i)}\right\} .
$$

Let $S_{0}$ be the "tree" generated by $x_{p_{0}}$.

Note that each $S_{m}$ is a subtree of $S$ and $S=\bigcup S_{m}$. Moreover, by the faithfulness of the $p_{m}$ 's, if $s \in S$ and $t \in S_{m}$ are incomparable, then there is $s^{\prime} \in S_{m}$ such that $s^{\prime} \subseteq s$ and $s^{\prime} \perp t$.

It follows that if $s$ is a maximal element of $S_{m}$, then there are at most $g\left(j_{m}\right)$ pairwise incomparable elements of $S_{m+1}$ extending $s$. 
Now let $G$ be $\mathbb{P}_{\geq n}$-generic with $q\left\lceil I_{\geq_{n}} \in G\right.$. Let $T$ be the tree generated by

$$
\left\{x_{r}: r \in \mathbb{P} \text { is compatible with each element of } G\right\} .
$$

Claim 4.5. $T$ is $<n$-ary.

Suppose not. Then there is $t \in T$ such that $\left|\operatorname{succ}_{T}(t)\right| \geq n$. Let $m \in \omega$ be minimal such that $\operatorname{succ}_{T}(t) \subseteq S_{m+1}$. By our observation above, $g\left(j_{m}\right) \geq n$. But for each $\sigma \in g\left(j_{m}\right)^{\eta_{m}\left(j_{m}\right)}$ exactly one of the conditions $q\left(j_{m}\right)_{\sigma-l}, l<g\left(j_{m}\right)$, is in $G$ (recall that we regard $\mathbb{Q}_{j_{m}}$ as a suborder of $\left.\mathbb{P}\right)$.

This means that only one of the sequences $x_{p_{m} *(\sigma-l)}, l<g\left(j_{m}\right)$, is contained in $T$. Therefore, for each maximal element $s$ of $T \cap S_{m}$ all the extensions of $s$ in $T \cap S_{m+1}$ are compatible. Since all sequences of the form $t \frown l, l \in \omega$, which are in $S$ are contained in $S_{m+1}$, this contradicts the assumption $\left|\operatorname{succ}_{T}(t)\right| \geq n$ and finishes the proof of Claim 4.5 .

To finish the proof of the lemma, let $\dot{T}$ be a $\mathbb{P}_{\geq n}$-name for $T$.

For a $\mathbb{P}$-name $\dot{a}$ let $\operatorname{supt}(\dot{a}) \subseteq I$ be minimal such that $\dot{a}$ is in fact a $\mathbb{Q}$-name where $\mathbb{Q}$ is the countable support product over $\left(\mathbb{Q}_{i}\right)_{i \in \operatorname{supt}(\dot{a})}$. Note that under $\mathrm{CH}$, $\mathbb{P}$ satisfies the $\aleph_{2}$-c.c. and thus every $\mathbb{P}$-name $\dot{a}$ for a real is equivalent to a $\mathbb{P}$-name $\dot{b}$ with $|\operatorname{supt}(\dot{b})| \leq \aleph_{1}$.

For $j \in I$ let $\dot{x}_{j}$ be the $\mathbb{Q}_{j}$-name for the generic real added by $\mathbb{Q}_{j}$, i.e., for the unique common branch of all conditions in a $\mathbb{Q}_{j}$-generic filter. We also regard $\dot{x}_{j}$ as a $\mathbb{P}$-name. For a tree $T$ let $[T]:=\left\{x \in \omega^{\omega}: \forall m \in \omega(x \uparrow m \in T)\right\}$.

Lemma 4.6. If $\dot{T}$ is a $\mathbb{P}$-name for $a<n$-ary tree and $j \in I \backslash \operatorname{supt}(\dot{T})$ is such that $g(j) \geq n$, then $1_{\mathbb{P}} \Vdash \dot{x}_{j} \notin[\dot{T}]$.

Proof. Let $p \in \mathbb{P}$. It suffices to find $r \leq p$ such that $r \Vdash \dot{x}_{j} \notin[\dot{T}]$.

Let $t \in p(j)$ be such that $\left|\operatorname{succ}_{p}(t)\right| \geq n$. Then for some $m \in \omega, t \in g(j)^{m}$. Since $j \notin \operatorname{supt}(\dot{T})$, there is $q \leq p$ such that $q$ decides $\dot{T} \cap \omega^{m+1}$ and $q(j)=p(j)$. Let $S \subseteq \omega^{m+1}$ be such that $q \Vdash S=\dot{T} \cap \omega^{m+1}$. By the assumption on $\dot{T}, S$ is $<n$-ary. Therefore, there is $l \in g(j)$ such that $t \frown l \notin S$. Let $r \leq q$ be such that all elements of $r(j)$ are comparable with $t \frown l$. Now $r \Vdash t \frown l \subseteq \dot{x}_{j}$ and thus $r$ is as required.

Lemma 4.3 and Lemma 4.6 enable us to prove:

Theorem 4.7. Let $M$ be a model of $G C H$ and let $f: \omega \backslash 2 \rightarrow\{\kappa \in$ Card $: \operatorname{cf}(\kappa)>$ $\left.\aleph_{0}\right\}$ be non-increasing. Let $\mathbb{P}$ be defined as above. If $G$ is $\mathbb{P}$-generic over $M$, then

$$
M[G] \models 2^{\aleph_{0}}=f(2) \text { and for all } n>2, \operatorname{cov}\left(\mathcal{I}_{n}\right)=f(n) .
$$

Proof. It is clear that $2^{\aleph_{0}}=f(2)$ in $M[G]$. Let $n>2$. Lemma 4.3 tells us that in $M[G]$ every $x \in \omega^{\omega}$ is a branch through a $<n$-ary tree which has a $\mathbb{P}_{\geq n}$-name. But up to equivalence, there are only $f(n) \mathbb{P}_{\geq n}$-names for subtrees of $\omega^{<\bar{\omega}}$. This shows $M[G] \models \operatorname{cov}\left(\mathcal{I}_{n}\right) \leq f(n)$.

On the other hand, in $M[G]$ let $\mathcal{F}$ be an uncountable family of $<n$-ary trees of size $<f(n)$. By the $\aleph_{2}$-c.c. of $\mathbb{P}$, there is a family $\mathcal{D}$ of $\mathbb{P}$-names for $<n$-ary trees of the same size such that each $T \in \mathcal{F}$ has a name $\dot{T} \in \mathcal{D}$. We may assume that $\left|\bigcup_{\dot{T} \in \mathcal{D}} \operatorname{supt}(\dot{T})\right|<f(n)$. Now there is $j \in I \backslash \bigcup_{\dot{T} \in \mathcal{D}} \operatorname{supt}(\dot{T})$ such that $g(j) \geq n$. It follows from Lemma 4.6 that $\left(\dot{x}_{j}\right)_{G}$ is not a branch through any of the trees in $\mathcal{F}$. This implies $M[G] \models \operatorname{cov}\left(\mathcal{I}_{n}\right) \geq f(n)$. 
4.3. $\operatorname{cov}\left(\mathcal{I}_{\omega}\right)$ can be smaller than $\operatorname{cov}\left(\mathcal{I}_{n}\right)$. It is possible to include $\operatorname{cov}\left(\mathcal{I}_{\omega}\right)$ in the statement of Theorem 4.7. The natural forcing notion corresponding to $\operatorname{cov}\left(\mathcal{I}_{\omega}\right)$ is the following:

Definition 4.8. Let $\mathbb{D}_{\omega}$ be the partial order consisting of all subtrees $p$ of $T_{\omega}$ such that for each $t \in p,\left|\operatorname{succ}_{p}(t)\right| \in\{1, \operatorname{dom}(t)+1\}$ and there is $s \in p$ such that $t \subseteq s$ and $\left|\operatorname{succ}_{p}(s)\right|=\operatorname{dom}(s)+1$.

$\mathbb{D}_{\omega}$ adds a new element of $\prod_{n \in \omega}(n+1)$ which is not contained in any element of $\mathcal{I}_{\omega}$ coded in the ground model. Let $f:(\omega+1) \backslash 2 \rightarrow\left\{\kappa \in \operatorname{Card}: \operatorname{cf}(\kappa)>\aleph_{0}\right\}$ be non-increasing.

Let $J:=\{\alpha \in \omega+1: \neg \exists \beta<\alpha(f(\beta)=f(\alpha))\}$. Let $I$ be a set of size $f(2)$ and let $g: I \rightarrow J$ be a function such that for each $\alpha \in J,\left|g^{-1}(\alpha)\right|=f(\alpha)$. For each $i \in I$ let $\mathbb{Q}_{i}:=\mathbb{D}_{g(i)}$. Finally, let $\mathbb{P}$ be the countable support product over the sequence $\left(\mathbb{Q}_{i}\right)_{i \in I}$.

Generalizing the proof of Theorem 4.7 we obtain:

Theorem 4.9. Let $M$ be a model of $G C H$ and let $f:(\omega+1) \backslash 2 \rightarrow\{\kappa \in$ Card : $\left.\operatorname{cf}(\kappa)>\aleph_{0}\right\}$ be non-increasing. Let $\mathbb{P} \in M$ be defined as above. If $G$ is $\mathbb{P}$-generic over $M$, then

$$
M[G] \models 2^{\aleph_{0}}=f(2) \text { and for all } \alpha \in(\omega+1) \backslash 3, \operatorname{cov}\left(\mathcal{I}_{\alpha}\right)=f(\alpha) .
$$

\section{Concluding Remarks AND OPEn PROBlems}

Combining Theorem 4.9, Theorem 2.2, and Theorem 2.8 we get:

Corollary 5.1. Let $V$ be an infinite-dimensional Hilbert space. For each $n \in \omega \backslash 2$ there is a closed set $X_{n} \subseteq \mathbb{R}^{n}$ and there is a closed set $X_{\omega} \subseteq V$ such that for every non-increasing function $f:(\omega+1) \backslash 2 \rightarrow\left\{\kappa \in \operatorname{Card}: \operatorname{cf}(\kappa)>\aleph_{0}\right\}$ it is consistent that for each $\alpha \in(\omega+1) \backslash 2, \gamma\left(X_{\alpha}\right)=f(\alpha)$.

Clearly, Theorem 1.1] follows from this. Since for all $n, \mathbb{R}^{n}$ embeds into every infinite-dimensional Hilbert space, we have:

Corollary 5.2. For each $n \in \omega$ it is consistent that for every infinite-dimensional Hilbert space $V,\left|\sigma_{\text {conv }}(V)\right|$ is at least $n$.

However, we believe that the results on $\left|\sigma_{\text {conv }}\left(\mathbb{R}^{n}\right)\right|$ and on $\left|\sigma_{\text {conv }}(V)\right|$ for infinitedimensional Hilbert spaces $V$ are not optimal yet.

Question 5.3. a) Let $n>2$. Is $\left|\sigma_{\text {conv }}\left(\mathbb{R}^{n}\right)\right|=n$ consistent?

b) Let $V$ be an infinite-dimensional Hilbert space. Can $\left|\sigma_{\text {conv }}(V)\right|$ be infinite?

If we want to construct a model in which $\left|\sigma_{\text {conv }}\left(\mathbb{R}^{n}\right)\right|=n$ for some $n>2$, the natural strategy is to find a model in which the cardinals $\operatorname{cov}\left(\mathcal{I}_{m}\right), m \in\{3, \ldots, n\}$, are strictly smaller than $\mathfrak{h m}$ and pairwise different and in which $\mathfrak{h m}$ is strictly less than $2^{\aleph_{0}}$. However, we do not know how to do this. We do not even know the answer to:

Question 5.4. Is $\aleph_{1}<\mathfrak{h m}<2^{\aleph_{0}}$ consistent?

A problem which is probably more of geometric than of set-theoretic nature is the problem of getting reasonable upper bounds for the size of $\sigma_{\text {conv }}\left(\mathbb{R}^{n}\right)$.

Question 5.5. Let $n>2$. Is $\left|\sigma_{\text {conv }}\left(\mathbb{R}^{n}\right)\right|$ finite? Is $\left|\sigma_{\text {conv }}\left(\mathbb{R}^{n}\right)\right| \leq n$ ? 


\section{REFERENCES}

[1] T. Bartoszynski, H. Judah, Set Theory: On the structure of the real line. A K Peters, Ltd., Wellesley, MA, 1995. MR 96k:03002

[2] A. Blass, Simple cardinal characteristics of the continuum. Set theory of the reals (Ramat Gan, 1991), 63-90, Israel Math. Conf. Proc., 6, Bar-Ilan Univ., Ramat Gan, 1993. MR 94i:03098

[3] S. Geschke, M. Kojman, W. Kubis, R. Schipperus, Convex decompositions in the plane and continuous pair colorings of the irrationals, submitted.

[4] M. Goldstern and S. Shelah, Many simple cardinal invariants of the continuum, Arch. Math. Logic 32 (1993), no. 3, 203-221. MR 94c:03064

[5] T. Jech, Multiple Forcing, Cambridge Tracts in Mathematics, 88. Cambridge University Press, Cambridge, 1986. MR 89h:03001

[6] L. Newelski, A. Roslanowski, The ideal determined by the unsymmetric game, Proc. Amer. Math. Soc. 117 (1993), no. 3, 823-831. MR 93d:03053

[7] S. Shelah, J. Steprans, The covering numbers of Mycielski ideals are all equal, J. Symbolic Logic 66 (2001), 707-718.

[8] J. Steprans, Decomposing Euclidean space with a small number of smooth sets., Trans. Amer. Math. Soc. 351 (1999), no. 4, 1461-1480. MR 99f:04002

[9] J. Zapletal, Isolating cardinal invariants, preprint.

Freie Universität Berlin, Arnimallee 2-6, D-1495 Berlin

E-mail address: geschke@math.fu-berlin.de

Department of Mathematics, Ben Gurion University of the Negev, Beer Sheva, Israel E-mail address: kojman@math.bgu.ac.il 